\title{
Prevalence of Diabetic Complications and Its Associated Factors Among Diabetes Mellitus Patients Attending Diabetes Mellitus Clinics; Institution Based Cross Sectional Study
}

\author{
Kidist Reba Lebeta ${ }^{1}$, Zeleke Argaw ${ }^{2}$, Bizuayehu Walle Birhane ${ }^{3}$ \\ ${ }^{1}$ Department of Nursing, College of Medicine and Health Science, Bahir Dar University, Bahir Dar, Ethiopia \\ ${ }^{2}$ Department of Nursing, College of Medicine, Addis Ababa University, Addis Ababa, Ethiopia \\ ${ }^{3}$ Department of Physiology, College of Medicine and Health Science, Bahir Dar University, Bahir Dar, Ethiopia
}

Email address:

kidistreba@gmail.com (K. R. Lebeta), zelekeargawm@yahoo.com (Z. Argaw), tezeramolla@gmail.com (B. W. Birhane)

\section{To cite this article:}

Kidist Reba Lebeta, Zeleke Argaw, Bizuayehu Walle Birhane. Prevalence of Diabetic Complications and Its Associated Factors Among Diabetes Mellitus Patients Attending Diabetes Mellitus Clinics; Institution Based Cross Sectional Study. American Journal of Health Research. Vol. 5, No. 2, 2017, pp. 38-43. doi: 10.11648/j.ajhr.20170502.13

Received: August 2, 2016; Accepted: February 22, 2017; Published: March 9, 2017

\begin{abstract}
Diabetes is a chronic disease, leading to many complications. Therefore the aim of this study was to determine the prevalence ofdiabetic complications and its associated factors. To provide evidence for this conjecture, Institution-based crosssectional study was conducted. Samples were chosen by systematic sampling technique. Data were collected by pretested questionnaire and document review; data were entered and analyzed by using SPSS version 20. Among 344 type 2 diabetes patients, more than half (53.5\%) of participants had major DM complications. Among major DM complications were diabetic retinopathy, diabetic foot ulcer and diabetic nephropathy with a prevalence of $25.5 \%, 21.2 \%$ and $11.4 \%$ respectively. In multiple logistic regressions Ages, duration of DM and drug regimen were significantly associated with DM complications. Prevalence of diabetes mellitus complication among the type 2 DM patient is high in study area. Therefore, the health care provider should strength early detection and treatment DM client.
\end{abstract}

Keywords: Diabetic Complications, Type 2 DM Clients, Amahara Regional State, Ethiopia

\section{Introduction}

Diabetes is one of the commonest and non-communicable disease remains highly prevalent with an increasing incidence globally. It is classified under three major groups, namely, type 1, type 2, and gestational diabetes [1]. World health organization defined diabetes mellitus (DM) as a metabolic disorder of multiple etiology characterized by chronic hyperglycemia with disturbances of carbohydrate, fat and protein metabolism resulting from defects in insulin secretion, insulin action, or both [2].

According to the International Diabetes Federation, Type 2 diabetes accounts for $85 \%$ to $95 \%$ of all diabetes in highincome countries and may account for an even higher percentage in low- and middle income countries. Type 2 diabetes is a common condition and a serious global health problem [1]. Type 2 diabetes mellitus is a multidimensional health problem with important consequences owing to its increasing prevalence, chronicity and complications that cause disability, decreased health-related quality of life (HRQOL) and premature death [3].

Diabetes is a chronic disease, leading to many complications include micro vascular (nephropathy, retinopathy, and neuropathy) and macro vascular (stroke, myocardial infarction, and coronary artery disease) with co morbidities leads to a substantial decrease in the patients' quality of life as well as socio-economic implications [4]. It is a chronic disease with considerable impact on health status and quality of life and it is considered an urgent public health issue because it has a pandemic potential [5].

The goal of this study is to determine diabetic complications and its associated factors. The findings of this 
study will assist health care professionals to understand factors related to diabetic complications and it also will help policy makers, program planning bodies and service providers to evaluate quality of existing policies, treatment strategies, programs and treatment guidelines and to improve or change them to attain optimum level of functioning and also helps as a baseline for future studies.

Finally since there is a limited research at country and lower level, this study can be used as resource for other studies related to complications in diabetes.

\section{Methods and Materials}

\subsection{Study Area and Period}

The study was conducted at FelegeHiwot Referral Hospital found in Bahir Dar which is capital city of Amhara regional state, located $565 \mathrm{kms}$ from Addis Ababa in North West of Ethiopia.

FelegeHiwot Referral Hospital is a tertiary health care level hospital serving the population of Bahir Dar town and remote areas of northwest Ethiopia. According to CEO (chief executive officer) office report, the total population served by the hospital is about 12 million. It is the only governmental hospital in the city and the regional referral hospital serving the population in the region as referral center. It has 200 beds and there are three medical OPD serves for medical patients of which one serves as referral clinic for patients with chronic diseases. Institutional based cross sectional design was conducted from April 15 to May 15, 2015during the routine working hours of the hospital.

\subsection{Sampling}

All type 2 diabetes patients who have follow-up at FelegeHiwot Referral Hospital were taken as a source population and the study population was those Sampled adult type 2 diabetes patients having follow-up at FelegeHiwot Referral Hospital during data collection period and those who full fill inclusion criteria. Known type 2 diabetes Patients having greater than or equal to one year follow up and aged 18 years and above used as an inclusion criteria.

Sample size was calculated using a single population proportion formula assuming proportion of $2 \mathrm{DM}$ patients who developed complication is $\mathrm{p}(0.5)$ since no previous study was conducted in study area, 5\% margin of error (d) and $95 \%(\mathrm{za} / 2=1.96)$ confidence level, Since the source population was 1,678 that was below 10,000 finite population correction was used we got the sample size 313, then considering non response rate of $10 \%$, the final sample size was 344

\subsection{Sampling Technique and Sampling Procedure}

A systematic random sampling was employed to select study participant. The diabetic clinic provides their service from Monday up to Friday every week. On average 183 patients with Type 2 DM are treated per week while 733 patients are treated per month. Based on the decision to collect data over the course of one month the sampling interval was determined by dividing the expected number of type 2 diabetic patients per month into the sample size (344) which gives approximately a sampling interval of two. Then the data was collected from each study participant with the interval of two until the desired sample size was reached.

\subsection{Data Collection Method}

The data from participants was collected using an interview -administered questionnaire and document review. The questionnaire was originally developed in English and then translated into Amharic. It was be then back translated to English by another person. The wording and sequence of questions were designed in such a way that the logical flow of ideas (from general to specific, from impersonal to personal, and from easy to difficult questions) will be maintained. Data was collected by three trained data collectors.

\subsection{Data Collection Procedure}

The aims, objectives, purpose of the study were orally explained to diabetic patients, who fulfill the inclusion criteria. Then the Data collectors were interview eligible and voluntary patients in a favorable place and the supervisors were monitored the data collection process.

\subsection{Data Processing and Data Quality}

The questionnaire were translated to Amharic and back translated to English by a translator who was blind to the original questionnaire. Pre-testing of the questionnaire were undertaken in 5 percent of the sample size in out of the study area, before the actual data collection were taken place and corrections on the instruments were made accordingly. A total of three days' intensive training were given for all supervisors and data collectors. Overall activities were controlled by the principal investigators.

\subsection{Data Management and Data Analysis}

The data was checked for completeness and consistency. Data cleaning was made by removing missing ideas and responses to questions about relevant information. Data entry and analyses were performed using SPSS statistical packages (version 20 for Windows). The odds of patient susceptibility to DM complication were examined using the odds ratio calculation at $95 \%$ confidence interval.

\subsection{Ethical Consideration}

Ethical clearance and approval to conduct the research was obtained from Addis Ababa University College of Health Science School of allied Health Science, department of nursing and midwifery research and Ethical committee. Official letter from department of nursing and midwifery was written to FelegeHiwot Hospital. In addition, informed consent was obtained from study participant to confirm their willingness for participation after explaining the objective of 
the study. Respondents were clearly told that they have the right to refuse or terminate at any point of the interview. Respondents was reassured that they would be anonymous.

\section{Result}

\subsection{Socio-Demographic Characteristics}

A total of 344 type 2 diabetes patients who had follow-up in DM clinic of FHRH were involved in the study to respond to interviewed questionnaire with the response rate of $100 \%$ (344). Among the total respondents, majority of them 197 $(57.3 \%)$ were male and more than $30 \%$ of them were $>47$ years old, with a mean age with standard deviation of $40.54 \pm 15.208$. As for marital status, more than half 209 $(60.8 \%)$ of the study participants were married and for educational level, majority $143(41.6 \%)$ had received secondary school education and above. Most of the study participants were government employee and unemployed with percentage distribution of 30.2 and 22.4 respectively (Table 1).

\subsection{Diabetes Mellitus Complication and Duration on DM Among the Study Participant}

More than half 184 (53.5\%) of the participants had major DM complications. Among major DM complications that are recorded from the study participant, the leading once are diabetic retinopathy, two and above complications, diabetic foot ulcer and diabetic nephropathy with a percentage distribution of $25.5 \%, 21.7 \%, 21.2 \%$ and $11.4 \%$ respectively. More than half 193 (57\%) of the study participant had been diagnosed with diabetes from 1 up to 5 years of duration. (Figure 1-3).

\subsection{Association Between DM Complications and Independent Variables}

Bivariate analysis was conducted to see the association between explanatory variables, such as gender, age, educational status, marital status, occupation, duration of DM and drug regimen with outcomes variable (DM complications). As Table 2 shows age, education level, marital status, duration of DM and drug regimens have association with DM complications.

Being above 47 years, 38-47 and 28-37 years have association with DM complications when compared with those whose age is $18-27$. [COR: $7.20,95 \% \mathrm{CI}(3.77-13.7)$ ], [COR: 3.35, 95\%CI (1.74-6.44)] and [COR: 2.16, 95\%CI (1.12-4.14)] respectively. Completing secondary school and above and primary school have association with DM complications when compared with those who can't read and write. [COR: $0.38,95 \% \mathrm{CI}(0.23-0.64)$ ] and [COR: 0.42, 95\%CI (0.22-0.78)] respectively. Being married, divorced and widowed have association with DM complications when compared with those who are single.[COR: 3.70, 95\%CI (1.58-8.62)], [COR: 2.78, 95\%CI (1.15-6.64)] and [COR: $1.80,95 \% \mathrm{CI}(1.00-3.20)]$ respectively. Living with DM for 6-10 years and above 10 years has association with DM complications when compared with living for 1-5 years. [COR: 10.43, 95\%CI (5.15-21.15)] and [COR: 5.00, 95\%CI $(2.75-8.82)]$ respectively. Being medicated with insulin only and insulin and oral anti DM medication have association with DM complications when compared with only oral anti DM medication [COR: 10.43,95\%CI (5.15-21.15) and [COR: $5.00,95 \% \mathrm{CI}(2.75-8.82)$ respectively. (Table 2$)$

\subsection{Factors Associated with DM Complications}

Bivariate analysis was conducted to see the association between explanatory variables, such as gender, age, educational status, marital status, occupation, duration of DM and drug regimen with outcomes variable (DM complications). Among those variables age, education level, marital status, duration of DM and drug regimens have association with DM complications. Then those associated variables entered in into multiple logistic regressions to identify factors associated with DM complications to control confounders. In the final model of the regression age, duration of DM and drug regimen were statistically significantly associated with DM complications after adjusting for other variables. DM patients whose age is above 47 years, 38-47 and 28-37 year are 6.17, 3 and 2.75 times more likely to develop DM complications than those in age group of 18-27 years. [AOR: $6.17,95 \% \mathrm{CI}:(2.52-15.09)]$, [AOR: 3.00, 95\% CI: (1.12-6.70)] and [AOR: 2.75, 95\% CI: (1.24-7.25)] respectively.

DM patients who live with DM above 10 years and between 6-10 years are 7.28 and 2.44 times more likely to develop DM complication than those who are living with DM between 1-5 years. [AOR: 7.28, 95\% CI: (3.32-16.00)] and[AOR: 2.44, 95\% CI: (1.26-9.34)] respectively. DM patients who medicated with insulin only and Insulin with oral anti DM medication are 4.2 and 3.70 times more likely to develop DM complication than those who medicated Oral anti DM medication only.[AOR:4.2, 95\% CI: (2.00-8.85)] and [AOR:4.2, 95\% CI:(1.46-9.37)] respectively. (Table 2)

\section{Discussion}

In the current study, the overall DM complications magnitude is $53.5 \%$. This figure is relatively similar with the study conducted in Australia and china where prevalence of DM Complications among Type 2 DM patients is 53\% and $52 \%$ respectively $[6,7]$. But, it is lower when compared with the study conducted in Benin where the prevalence of DM complications is $78 \%$. This big difference may be due to exclusion of individuals whose age is less than 25 years in that of Benin [8].

Retinopathy is the leading complication accounting $25.5 \%$ out of overall DM complication. This finding is almost similar with study conducted in India and Australia where the prevalence of retinopathy is $24.5 \%$ and $26.2 \%$ respectively $[9,10]$. In present study diabetic foot ulcer covers $21.1 \%$ of the DM complication which is the same with study conducted to assess risk factor for foot ulcer where $21.6 \%$ of the participant developed diabetic foot ulcer [6]. The diabetic 
nephropathy covers $11.4 \%$ out of overall DM complication which also almost the same with study conducted in china where prevalence of nephropathy is $10.7 \%$ [7]. But there is difference in prevalence when compared with that of the study conducted in Benin with prevalence of nephropathy $20 \%$. This difference may be due to exclusion of individuals whose age is less than 25 years and using of small sample size in that of Benin [8].

In this study, DM complication is significantly associated with Age, duration of living with DM and drug regimen. Older DM patients whose age is 47 years, 38-47 and 28-37 year are $6.17,3$ and 2.75 times more likely to develop DM complications than those in age group of 18-27 years respectively. Different research findings also show significant association between older age and DM complication [6-12]. But, in study conducted among Australian population age have no effect on DM complication. The difference may be due to focusing of the study among Australian population is only on retinopathy complication and its being population based study [9]. DM patients who live with DM above 10 years and between 6-10 years are 7.28 and 2.44times more likely to develop DM complication than those who are living with DM $<5$ years respectively. This significant association of DM complication withduration of living with DM is similar to that found in other studies [6-12]. DM patients who medicated with insulin only and Insulin with oral anti DM medication are 4.2 and 3.70times more likelyto develop DM complication than those who medicated Oral anti DM medication only respectively. This finding is supported by study conducted among U.S. veterans to assess the risk of developing a diabetic foot ulcer where insulin is independent predictor for developing foot ulcer [11].

\section{Conclusion and Recommendation}

In conclusion, Diabetes mellitus complication among the type $2 \mathrm{DM}$ patient is high in study area. The variation level of DM complications is well explained by age, duration of DM illness and drug regimen. Therefore, the health care provider should strength early detection and treatment of those DM clients. Screening and treating physicians should be encouraged to exert more attention and care to eye examination and foot examination, especially among the elderly, long duration of DM illness and took insulin medications; as ample evidence from the study proves that potential risk factors for DM complications are highly significantly associated with age, duration of illness and drug regimen.

Table 1. Frequency distribution of respondents' Socio-demographic characteristics of type 2 diabetic patient in FelegeHiwot Referral hospital, Bahir Dar, 2015.

\begin{tabular}{|c|c|c|}
\hline Variable & Frequency (N) & Percentage $(\%)$ \\
\hline \multicolumn{3}{|l|}{ Gender } \\
\hline Male & 197 & 57.3 \\
\hline Female & 147 & 42.7 \\
\hline \multicolumn{3}{|l|}{ Age in year } \\
\hline $18-27$ & 83 & 24.1 \\
\hline $28-37$ & 77 & 22.4 \\
\hline $38-47$ & 78 & 22.7 \\
\hline$>47$ & 106 & 30.8 \\
\hline \multicolumn{3}{|l|}{ Educational status } \\
\hline Can't read \& write & 117 & 34 \\
\hline Read \& write but no formal educ. & 23 & 6.7 \\
\hline Primary education & 61 & 17.7 \\
\hline Secondary education and above & 143 & 41.6 \\
\hline \multicolumn{3}{|l|}{ Religion } \\
\hline Orthodox & 267 & 77.6 \\
\hline Muslim & 70 & 20.3 \\
\hline Protestant & 7 & 2.1 \\
\hline \multicolumn{3}{|l|}{ Marital status } \\
\hline Single & 62 & 18 \\
\hline Married & 209 & 60.8 \\
\hline Divorced & 33 & 9.6 \\
\hline Widowed & 40 & 11.6 \\
\hline \multicolumn{3}{|l|}{ Occupation } \\
\hline Government employee & 104 & 30.2 \\
\hline Unemployed & 77 & 22.4 \\
\hline Merchant & 48 & 14.0 \\
\hline Student & 22 & 6.4 \\
\hline Farmer & 76 & 22.1 \\
\hline Private employee & 17 & 4.9 \\
\hline Income (Mean \& S. D)ETB & $1962.62 \pm 2232.873$ & \\
\hline
\end{tabular}

Table 2. Factors associated withDM complication among DM Patients Attending DM Clinics ofFelegeHiwot Referral Hospital, Bahir Dar, 2015, (n=344).

\begin{tabular}{|c|c|c|c|c|}
\hline \multirow{2}{*}{ Variables } & \multicolumn{2}{|c|}{ DM complication } & \multicolumn{2}{|l|}{ OR 95\% CI } \\
\hline & Yes & No & Crude & Adjusted \\
\hline \multicolumn{5}{|l|}{ Agein years } \\
\hline $18-27$ & 24 & 59 & 1 & 1 \\
\hline $28-37$ & 36 & 41 & $2.16(1.12-4.14)^{*}$ & $2.75(1.24-7.25) *$ \\
\hline $38-47$ & 45 & 33 & $3.35(1.74-6.44)^{*}$ & $3.00(1.12-6.70) *$ \\
\hline$>47$ & 79 & 27 & $7.20(3.77-13.7)^{*}$ & $6.17(2.52-15.09) *$ \\
\hline \multicolumn{5}{|l|}{ Educational status } \\
\hline Read \& write & 7 & 16 & $1.14(0.43-3.0)$ & $0.67(0.22-2.14)$ \\
\hline Primary education & 33 & 28 & $0.42(0.22-0.78)^{*}$ & $0.81(0.34-1.90)$ \\
\hline Secondary and above & 81 & 62 & $0.38(0.23-0.64)$ & $0.62(0.28-1.40)$ \\
\hline \multicolumn{5}{|l|}{ Marital status } \\
\hline Single & 24 & 38 & 1 & 1 \\
\hline Married & 111 & 98 & $1.80(1.00-3.20)^{*}$ & $0.86(0.38-1.92)$ \\
\hline Divorced & 21 & 12 & $2.78(1.15-6.64) *$ & $0.88(0.28-2.78)$ \\
\hline
\end{tabular}




\begin{tabular}{|c|c|c|c|c|}
\hline \multirow{2}{*}{ Variables } & \multicolumn{2}{|c|}{ DM complication } & \multicolumn{2}{|l|}{ OR 95\% CI } \\
\hline & Yes & No & Crude & Adjusted \\
\hline Widowed & 28 & 12 & $3.70(1.58-8.62)^{*}$ & $1.22(0.38-3.90)$ \\
\hline \multicolumn{5}{|l|}{ Occupation } \\
\hline Government employee & 50 & 54 & 1 & 1 \\
\hline Unemployed & 39 & 38 & $1.10(0.62-1.20)$ & $1.30(0.53-3.19)$ \\
\hline Merchant & 21 & 27 & $0.84(0.42-1.67)$ & $1.03(0.442-2.35)$ \\
\hline Student & 9 & 13 & $0.74(0.23-1.90)$ & $3.16(0.91-10.95)$ \\
\hline Farmer & 53 & 23 & $2.49(1.33-4.64)^{*}$ & $1.90(0.72-4.96)$ \\
\hline Private employee & 12 & 5 & $2.56(0.85-7.88)$ & $2.60(0.66-10.20)$ \\
\hline \multicolumn{5}{|l|}{ Duration of DM } \\
\hline $1-5$ years & 68 & 128 & 1 & 1 \\
\hline $6-10$ years & 61 & 11 & $10.43(5.15-21.15)^{*}$ & $2.44(1.26-9.34) *$ \\
\hline$>10$ years & 55 & 21 & $5.00(2.75-8.82)^{*}$ & $7.28(3.32-16.00) *$ \\
\hline \multicolumn{5}{|l|}{ Drug Regimen } \\
\hline Oral anti DM medication only & 18 & 56 & 1 & 1 \\
\hline Insulin only & 142 & 79 & $5.60(3.07-10.17)^{*}$ & $4.20(2.00-8.85) *$ \\
\hline Insulin \& oral anti DM medication & 24 & 25 & $2.98(1.38-6.46) *$ & $3.70(1.46-9.37) *$ \\
\hline
\end{tabular}

$*=$ significant at $\mathrm{p}$-value $<0.05$

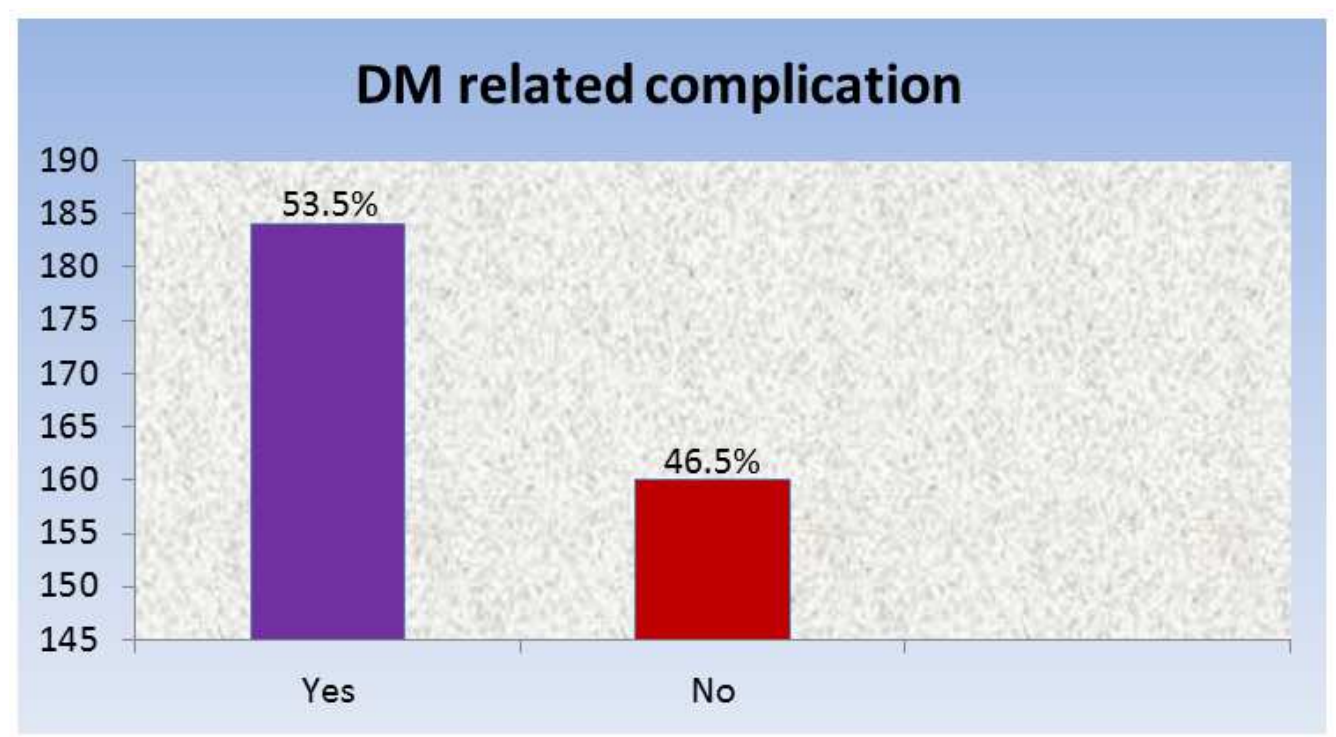

Figure 1. Distribution of DM related complications among DM Patients Attending DM Clinics ofFelegeHiwot Referral Hospital, Bahir Dar, 2015.

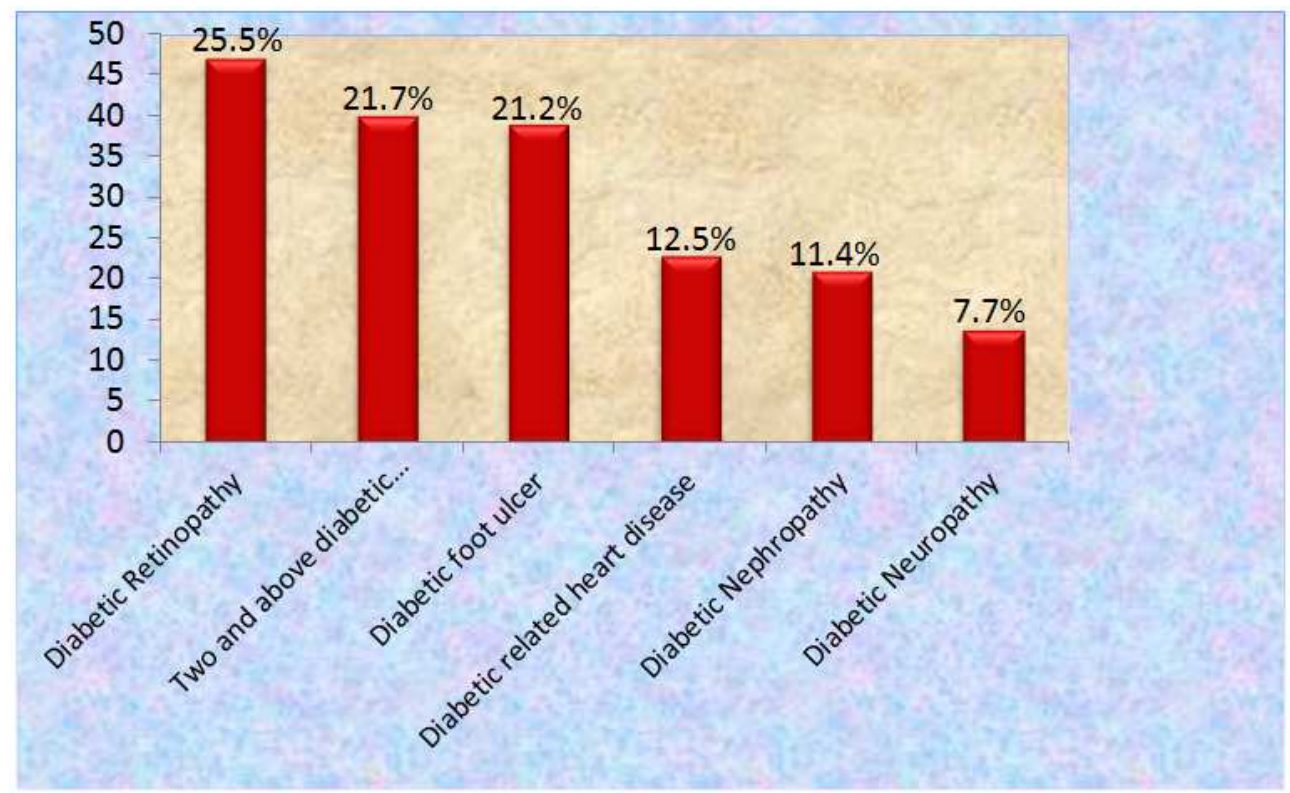

Figure 2. Distribution of major DM related complications among DM Patients Attending DM Clinics of FelegeHiwot Referral Hospital, Bahir Dar, 2015. 


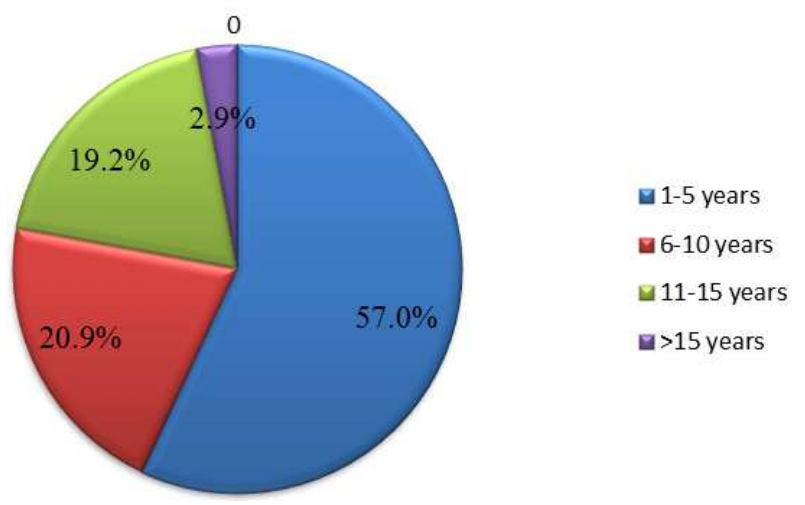

Figure 3. Distribution of duration of type 2 diabetes illness DM Patients Attending DM Clinicsof FelegeHiwot Referral Hospital, Bahir Dar, 2015.

\section{References}

[1] International Diabetic Federation diabetes atlas in IDF Atlas of DM, 6th, Editor. 2013.

[2] World Health Organization Department of Non communicable Disease Surveillance Geneva 1999.

[3] Zhang P ZX, Brown J et al. Global healthcare expenditure on diabetes for 2010 and 2030.. Diabetes Res ClinPract. 2010; 87: 293-301.

[4] Fal AM JB, Uchmanowicz I, Sen M, Panaszek B, Polanski J. Type 2 diabetes quality of life patients treated with insulin and oral hypoglycemic medication. ActaDiabetol 2011; 48 (3): 237-42.

[5] Boyle JP, Honeycutt AA, Narayan KV, Hoerger TJ, Geiss LS, Chen H, et al. Projection of Diabetes Burden Through 2050 Impact of changing demography and disease prevalence in the US. Diabetes Care. 2001; 24 (11): 1936-40.

[6] Davis WK. Psychosocial correlates of survival in diabetes. survival. 1988; 2: 3.

[7] Zhaolan Liu and et. al. Prevalence of chronic complications of type 2 diabetes mellitus in outpatients - a cross-sectional hospital based survey in urban China. 2010; 10 (8): 233-77.

[8] François Djrololand et. al. Diabetes Complications and Associated Factors in Type 2 Diabetic Patients in Cotonou. 2014; 19 (8): 217-84.

[9] Robyn J. Tapp and et. al. The Prevalence of and Factors Associated With Diabetic Retinopathy in the Australian Population. 2003; 28 (9): 123-64.

[10] Diabetic retinopathy among self reported diabetics in southern India: a population based assessment: V Narendran, R K John, A Raghuram, R D Ravindran, P KNirmalan, R D Thulasiraj, 2015; 32 (8): 27-14.

[11] Edward J. and et. al. A Prospective Study of Risk Factors for Diabetic Foot Ulcer, The Seattle Diabetic Foot Study. 1999; 19 (4): 267-43.

[12] Song SH. Complication characteristics between young-onset type 2 versus type 1 diabetes in a UK population. BMJ Open Diabetes Research and Care. 2015; 5 (4): 67-74. 